\title{
TRANSLATE ALS KOGNITIVE ENTITÄTEN ODER DIE NATÜRLICHKEITSTHEORIE IM KONTEXT DER SPRACHLICHEN UND PSYCHOSOZIALEN PARADIGMATA DER MEHRSPRACHLICHEN KOMMUNIKATION
}

\subsection{Einleitung}

Jahrtausende alt ist das Gefühl bzw. - gepaart mit Überlegungen und Reflexionen - die spätere Überzeugung von Philosophen, Literaten und Denkern, dass der ins Wort gefasste Gedanke den Gedanken selbst beeinflusst, verändert, ihm zu einer neuen, wenn auch vergleichbaren Existenz verhilft, aber dennoch den intendierten Sinn in entsprechender Kombination mit allen möglichen - auch nichtsprachlichen - Faktoren $\mathrm{zu}$ vermitteln und an Menschen zu transportieren vermag, die uns schon dadurch mehr oder weniger nahe stehen, dass sie die natüliche, soziale und kulturelle Umwelt mit uns teilen, aber unter gewissen Voraussetzungen auch an jene, die anderen Kulturkreisen, Religionen, ja Welten (und sei es nur im Sinne von Generationen) angehören.

Transferiert und mutiert durch Gedanken, Gefühle und Erfahrungen einzelner Menschen, sozialer Gruppen und Gemeinschaften oder ganzer Generationen verbreitet sich der Gedanke im ständigen Wandel von Konventionen, Sprachen und Kulturen. Vergleichbar mit einem realen oder fiktiven Universum ist er in Sprache gefasst in seinem Wesen allen zugänglich (universell), jedoch gleichzeitig von Fall zu Fall unterschiedlich als Eigentum der jeweiligen Kultur, Glaubens- oder Sozialgemeinschaft, ja sogar des einzelnen Menschen, gefärbt von dessen eigener, persönlicher Lebensgeschichte, die jedoch von der genetischen Geschichte des Menschen aufgenommen wurde als das kollektive, in die genetische Struktur gefasste Gedächtnis der Menschheit, das sich durch die Evolution von Beginn an bis heute entfaltet hat. Kein Erlebnis, kein jemals gedachter Gedanke geht je endgültig unter; vielmehr bildet er die Basis für weitere Veränderungen, für Wandel, Ausbreitung, Bereicherung, aber auch Schwund und Untergang, wenn er sich im Sinne Darwins als nicht lebensfähig erwies bzw. für den ewigen Kreislauf des Lebens - des Wortes - unter Umständen sogar störend hätte sein können.

Mit dieser etwas poetisch anmutenden Einleitung in die nüchterne Materie der Linguistik wollte ich eigentlich nur darauf verweisen, in welch vielfältiger Weise das komplexe System Sprache an alle Modalitäten des menschlichen Lebens - sei es seiner Geschichte oder seiner Gegenwart - verpflichtend gebunden ist. Diese komplexen Verbindungen ergeben sich schon unter Angehörigen einer Sprach- und Kultur gemeinschaft, geschweige denn, wenn es darum geht, die in einer Sprache festgehaltenen Intentionen in andere Sprach- bzw. Kultursysteme umzusetzen bzw. - in den Worten Andrew Chestermans (Chesterman, 2000) - in den Kulturkreis des Zielpublikums auszubreiten. 
1.0 Damit habe ich nun die mittelnde oder vermittelnde komplexe Tätigkeit des translatorischen Handelns (Übersetzen/Dolmetschen) zur Sprache gebracht und gleichzeitig Platz eingeräumt für jene Sprachtheorien, die über das sprachliche System hinaus denken, es in die natürliche, kulturelle und soziale Umwelt einbinden sowie mit den psychosozialen Gegebenheiten seiner Benutzer in Relation bringen. Funktionalismus, kognitive Theorien und die Natürlichkeitstheorie (konkret: die slowenische Theorie der sprachlichen Natürlichkeit: STSN) bilden in der Tat jenen theoretischen Rahmen des gegenwärtigen Aufsatzes, der die Grundlage darstellen wird für die Suche nach Berührungspunkten und gemeinsamen Erklärungsansätzen, aber auch Divergenzen zum Thema mehrsprachige Kommunikation.

2.0 Über den gemeinsamen Nenner der Informationsmittlung wird auf die Kernfrage der sprachlichen Verarbeitung und Speicherung von für kommunikative Zwecke verwendbaren Propositionen eingegangen. Um das Thema weiter zu führen und die Arbeitshypothesen aufstellen zu können, werden vordergründig die Erklärungsmodelle der Systemtheorie, wie sie in der kognitiven Linguistik Anwendung findet, und des STSN bemüht. Gibt es Überlappungen, wenn ja, worauf sind sie zurückzuführen und inwiefern verschärft sich durch das oben angedeutete spezielle Wissen die Optik für den Beobachter des sprachlichen / kommunikativen Handelns? Kann auf diese Weise auch in translatorische Probleme mehr Klarheit gebracht werden?

3.0 Auf die o.a. Fragenkomplexe wird in der weiteren Folge unter Beruicksichtigung konkreter translatorischer Handlungen innerhalb des slowenischdeutschen und deutsch-slowenischen Sprachenpaares eingegangen.

4.0 Den ersten Berührungspunkt zwischen den obgenannten Theorien finden wir in der ihnen gemeinsamen Behauptung, dass es sich bei allen die Kommunikation mitgestaltenden Ebenen um natürliche und interagierende Systeme handelt (Kognition, Sprache, Kultur).

5.0 Die kognitive Linguistik (Langacker1991, 1999; Rickheit-Strohner 1993; Karpf 1990) sieht ihren Ausgangspunkt in der Informationsverarbeitung. Im Mittelpunkt steht der Mensch mit seinem (auch sprachlichen) Eingreifen in die Umwelt zum Zweck seines (besseren) Überlebens, vor allem im Sinne seiner sozialen und kulturellen, aber auch biologischen Bedürfnisse. Indem der Mensch seine Umgebung mit seinen Sinnen wahrnimmt, verändert (modifiziert) er sie zugleich auch mit seinem bereits vorhandenen Weltwissen und seiner sprachlichen Kompetenz (Wahrnehmung als Erfindung = KONSTRUKTIVISMUS). Somit greift er auch mit der Sprache gestaltend in seine Umwelt ein, indem er die kommunikativen Ziele verwirklicht. Die Welt, auf die sich die beiden natürlichen Systeme Sprache und Kognition beziehen, ist demnach prinzipiell für alle Benutzer natürlicher Sprachen in ihren wesentlichen Zügen vergleichbar - in dieser inhärent gegebenen universellen Komponente liegt die Übersetzbarkeit von Sprachen überhaupt begründet -, gleichzeitig ist sie aber typologisch 
Sprachgruppen, Sprachfamilien) und individuell (Einzelsprachen) unterschiedlich.

5.1 Auch die Natürlichkeitstheorie (NT) geht vom Universellen, Prototypischen und Einzelsprachspezifischen aus, wobei alle Ebenen sowohl mit der Umwelt als auch untereinander interagieren, um neue, effiziente Kommunikationsmöglichkeiten zu schaffen, sich anzupassen und/oder zu verändern. Dabei zeichnen sich vor dem Hintergrund der Umwelt universelle Eigenschaften beider Systeme ab.

5.2 Die NT (Orešnik 1994, 1999, 2000, 2003, 2004; Mayerthaler 1983, 1987; Wurzl 1987, Dressler 1987, 1990; Teržan Kopecky 1997, 1998, 2001) arbeitet mit folgenden Postulaten:

* Hoher kognitiver Aufwand resultiert in hohen Markiertheitsstufen seiner Verbalisierung ((morpho)syntaktischer Konstruktion). Die natürliche und erwartbare Folge einer solchen Konstellation ist eine niedrige Token- und Typen-Frequenz und im Vergleich zu weniger markierten Konstruktionen, die auch weniger komplexe funktionale (kognitive) Komponenten transportieren, ein niedrigerer Grad der $\mathrm{Na}$ tiirlichkeit.

* Evolutionsmäßig, im Sinne der kategorialen Genese und den dabei durchlebten Grammatikalisierungsphasen, kann die Steigerung der Token- und Typen-Frequenz einer M+Konstruktion ein Hinweis sein auf die Verringerung ihrer Markiertheitswerte bei gleichzeitiger Steigerung ihres Natürlichkeitsgrades (die Konstruktion wird üblich und ist bereits eine typische Konstituente des jeweiligen grammatischen Systems).

* Der Natürlichkeitsgrad von Konstruktionen steht in einer Abhängigkeitsbeziehung zum jeweiligen Alter der Konstruktion. In diesem Sinn spricht man von Grammatikalisierungsphasen, wobei die späte oder Postgrammatikalisierungsphase durch Frequenzsteigerung und Markiertheitsreduzierung signalisiert wird. Die Letztere bezieht sich im STSN auf die Markiertheitsgrade des mikrogrammatischen Umfeldes von Konstruktionen.

Eine spezielle Ausprägung der STSN ist als Resultat statistischer, im Sinne der Korpuslinguistik angelegter Auswertungen umfangreicher deutscher und slowenischer Korpora (darunter auch zahlreiche Übersetzungstexte) als Repräsentanten unterschiedlicher Entwicklungsperioden von untersuchten grammatischen Kategorien (Grammatikalisierungsphasen) aber auch Textsorten erarbeitet worden. Diese Auswertung hat die Generierung des folgenden Postulats herbeigeführt:

STSN: $\mathrm{M}+=\mathrm{MGK}+$ (= relativ hohe Markiertheitsgrade von Konstruktionen werden in der Regel /im natürlichsten Fall von relativ hohen Markiertheitsstufen des mikrogrammatischen Kontextes begleitet (Reaktion des mikrogrammatischen Kontextes auf die Komplexität/Natürlichkeit der Konstruktion))

* Frequenzsteigerung und Markiertheitsreduzierung können auch vom Auftreten der komplexen syntaktischen Konstruktion in für sie natürlicheren Textsorten ausgelöst werden. Auch hier ist, vor allem in frühen Grammatikalisierungsphasen, 
ein Ausgleich der Markiertheit des Kontextes und der Konstruktion der Normalfall. Erst die Steigerung der Token-Frequenz und das zunehmende Alter der Konstruktion ebnen den Weg zu weniger komplexen textuellen Welten.

Das oben Vorgeführte zeigt, dass auch die angesprochene STSN die Sprache als ein natürliches, mit der sozialen und kulturellen Umgebung interagierendes und stark an die kognitiven Prozesse gebundenes System betrachtet.

6.0 Die kognitive Theorie geht davon aus, dass für die Erkennung/Verarbeitung von Informationen ein integrales System zur Verfügung steht, dessen Effizienz sich aus dem Zusammenwirken seiner Komponenten ergibt, wodurch die zwei Welten in ein unteilbares Ganzes verschmelzen: die philogenetische (Erbmasse; durch neuronale Vernetzungen vollzogene Filterung von eingelangten Informationen, ihre Analyse und Gruppierung zu Modulen; genetisch bedingte Arbeitsverfahren des Neocortex als des zentralen Prozessors) und die ontogenetische (Interaktion mit der Umwelt, Einknüpfung von psychosozialen Paradigmen in die Erkennungsprozesse). Bei den angesprochenen Komponenten (vgl. Petri 1994; Kess 1992; Karpf 1990, Karpf, Dringel-Techt 1995) des kognitiven Systems handelt es sich um

a/ die Information und den zentralen Prozessor (Hirnrinde mit ihren integrierenden Funktionen, die bereits von den internalisierten, aus der sozialen Umwelt aufgenommenen Informationen mitbestimmt sind und das s.g. Weltwissen konstituieren: unter anderem Kommunikationskonventionen, Eigenarten des Textproduzenten und des Textrezipienten, ihre Erwartungshaltun gen, Verhaltensverpflichtungen, usw.)

b/ Umwelt der beiden Zentralkomponenten (Objekte, die mit ihnen funktional verbunden sind)

c/ Struktur (Relationen zwischen den Komponenten)

d/ Funktion (Beziehung des Systems zur Umwelt)

Die oben angeführten Komponenten des natürlichen Systems Kognition erzeugen das Verhalten des Systems, in welchem sich Zustände, vor allem aber auch konstante Veränderungen abzeichnen.

6.1 Die Veränderungen zeigen sich als Dynamik des kognitiven Systems, was gleichzusetzen ist mit Veränderung von Zuständen in der Zeit unter gleichzeitiger Auswirkung auf das Verhalten des Systems (Rückwirkung) (Langacker 1991, 1999; Rickheit-Strohner 1993 u.a.). Die die Dynamik erzeugenden Systemkomponenten zerfallen im Wesentlichen in zwei Gruppen: Die autonomen Teilsysteme erzeugen ihre Dynamik bzw. die Veränderung des (K)Systems ohne Abstimmung mit der Umwelt; die interaktiven Teilsysteme stimmen sich hingegen mit der Umwelt ab. Beide kann man als Funktionen des ARBEITSGEDÄCHTNISSES (Aktivierung 
nach Wichtigkeit und Häufigkeit der bereits erfolgten positiven Aktivierung)

sehen. Somit kann man auch sprachliche Veränderungen zumindest teilweise als Folgen der spezifischen Arbeitsweise des Gedächtnisses auffassen, welches selbst als eine phylogenetische Gegebenheit entscheidend an der Gestaltung der natürlichen Systeme (wie Sprache und Kognition) mitwirkt, indem es an der so genannten Selbstorganisation (vgl. Karpf 1990), in deren Rahmen es als zentraler Prozessor in ständiger Wechselwirkung mit der Umwelt steht, maßgeblich mitbeteiligt ist.

\subsection{Entscheidend für die Erzeugung der Systemdynamik sind folgende Prozesstypen:}

A/ Die Steuerung als Basisprozess der Informationsverarbeitung bedeutet umgesetzt in die Kommunikationssituation, dass der Textproduzent die Zustände und das Verhalten des Textrezipienten steuert.

B/ Die Regelung sorgt für die Wiederherstellung eines Zustandes bzw. seine Beibehaltung trotz Einwirkungen der Umwelt. Es handelt sich dabei um ein besonders ausgeklügeltes Gestaltungsprinzip, das eigentlich für die GEGENWEHR DES SYSTEMS und seine STABILITÄT zuständig ist. Es verhindert die „Überdosierung“ der Einwirkungen der Umwelt auf das KSystem und funktioniert als eine Art Kontrollsystem zur Erhaltung des minimalen Gleichgewichts der Zustände des sprachlichen und des kognitiven Systems.

6.2.1 Hier ist zum Beispiel jene „Notbremse“ angesiedelt, die verhindert, dass z.B. die Abschwächung der Endsilben die Wurzelsilben „,angreift“, was Wörter zu Silben zurückführen würde, und die meines Erachtens unter anderem auch dafür sorgt, dass der sprachliche Innovationsprozess nicht ausschließlich die Richtung Markiertheitsabbau einschlägt, sondern des öfteren auch die Markiertheitsumkehr beobachtbar ist, was für das systeminterne Gleichgewicht sorgt. Aus diesem Grund wird diesem Prozesstyp im gegenwärtigen Aufsatz die meiste Aufmerksamkeit gewidmet, da zum einen gerade hier ein relevanter Verknüpfungspunkt mit der STSN angesiedelt ist, und zum anderen üblicherweise dieser Verarbeitungsebene weniger Aufmerksamkeit geschenkt wird, weil sie ausgesprochen diskret wirkt.

6.2.1.1 Die folgenden Beispiele aus empirischen Untersuchungen zur o.a. natürlichen/universellen Gesetzmäßigkeit, die man bei $\mathbf{L}$-, bei $\mathbf{K}$-, aber auch bei $\mathbf{T}$ (ranslations)-Prozessen beobachten kann, veranschaulichen auch die unter Punkt 5.2. vorgestellten Postulate. Sie beziehen sich speziell auf die Markiertheitsverhältnisse zwischen Ausgangs- und Zieltexten im 
Rahmen der aufgenommenen und ausgewerteten sprachlichen Handlungen des konsekutiven Dolmetschens (mehrerer von der Autorin aufgezeichneter und statistisch bearbeiteter Ereignisse $\mathrm{zu}$ unterschiedlichen Zeiten und Themenschwerpunkten). Gleichzeitig geben die unten angefülhrten statistischen Daten Aufschluss über die übereinzelsprachlichen (universellen), aber auch prototypischen grammatischen Parameter, die sich auch von den sonstigen kulturell und sozial bedingten L-spezifischen Erscheinungen, die gerade bei Translationsprozessen besonders gravierend in den Vordergrund treten, dadurch abheben, dass die Unterschiede durch die regelnde Tätigkeit des Sprachsystems größtenteils getilgt werden explizit an jenen Stellen des grammatischen Systems, wo sich universelle Prinzipien der NT geltend machen.

6.2.1.2 Von den aufgenommenen und transkribierten Texten, entstanden bei unterschiedlichen Handlungen des Konsekutivdolmetschens mit folgenden Variablen (Thema, ZT-Dt.; ZT-Sl; AT-Produzenten), wurden folgende, übereinzelsprachlich als konstant angenommene (= Konstituenten der universellen sprachlichen Ebene) Parameter untersucht. Die Vergleiche des Verhaltens der mikrogrammatischen Konstellation zeigten bei untersuchten grammatischen Parametern mit Ausnahme der typologisch bedingten Distributionseigenheiten keine relevanten Abweichungen in folgenden Fällen (AT(sl/dt) - ZT(sl/dt):

\title{
- Zahl der gebrauchten Nebensätze
}

(ist offensichtlich eine nicht typologische oder L-spezifische Eigenschaft)

\section{- analytische \\ Konstruktionen \\ $(40 \% \mathrm{sl}: 60 \% \mathrm{dt})$}

(Die geringere Abweichung zu Gunsten des Deutschen ist offensichtlich Lspezifisch bedingt (= das Dt. als bevorzugt analytische, das Sl. eine vorwiegend synthetische Sprache = hypothesenfreundliches Resultat.)

\section{- Auftretenshäufigkeit des Präsens \\ 70\% (übrige $\mathrm{T} 30 \%$ ) \\ $\mathrm{M}+$ Kollok. $=35 \%$}

(Es handelt sich um eine hypothesenfreundliche Gegebenheit im Sinne der STSN, weil das im Tempus-Paradigma weniger markierte Präsens generell (übereinzelsprachlich) höhere Token-Frequenzen aufweist, jedoch relativ niedrigere Markiertheitsstufen der kollozierenden grammatischen Parameter bevorzugt.)

\author{
Vergangenheitstempora \\ $15 \%$ \\ (davon $40 \%$ in NS)
}


(Auch hier zeigt sich eine hypothesenfreundliche Gegebenheit im Sinne der STSN, weil die im Tempusparadigma relativ markierteren Vergangenheitstempora erwartungsgemäß eine niedrigere token-Frequenz haben als die präsentischen Tempora, dafür aber in stärkerem Maße die Umgebung von komplexeren grammatischen Parametern präferieren, wie etwa die Nebensätze (NS).)

\section{W+I-Futur \\ $7 \%$ \\ $60 \%$ in NS}

(Auch beim analytischen Futur zeigten sich hypothesenfreundliche Resultate im Sinne der STSN (sowohl in den Ziel- als auch in den Ausgangstexten). Sie weisen nämlich als markiertere Konstruktionen noch niedrigere tokenFrequenzwerte auf als die Vergangenheitstempora, dafür steigt ihre Kollokationstendenz mit den komplexeren Nebensätzen um einen beträchtlichen Prozentsatz an.)

\section{$\mathrm{PF}$ \\ $2 \%$ \\ $45 \%$ in NS}

(Noch niedriger ist die token-Frequenz bei stark markierten Belegen des Präsens pro Futuro, das als nicht-präsupponierte Struktur (vor allem im Slowenischen) im Schnitt beider Sprachen die niedrigste Frequenz aufweist, dazu eine relativ hohe Kollokation mit markierteren Nebensätzen. Man würde in diesem Fall zwar noch höhere Präferenzen des Nebensatzes erwarten, was aber m.E. durch den diesbezüglichen typologischen Unterschied zwischen dem Deutschen (im Deutschen sind die PF-Belege weitaus üblicher als im Slowenischen) und dem Slowenischen verursacht wird.)

6.2.1.2.1Untersucht wurden auch die Auftretenshäufigkeiten des PASSIVS als einer komplexen syntaktischen Konstruktion, die relevante Abweichungen zwischen AT (Ausgangstext) und ZT(Zieltext) ausweisen:

$$
\begin{aligned}
& \text { ZTsl }=1,5 \% \\
& \text { ZTdt }=3,0 \%
\end{aligned}
$$

(Die Ursache dieses Ergebnisses ist sprachtypologischer Art, da das Deutsche die Passivkonstruktionen typologisch bedingt bevorzugt.)

Die token-Frequenz des Passivs bzw. seine Distribution auf Nebensätze (NS) in den Ausgangs- und Zieltexten zeigt das folgende Bild:

$$
\mathrm{AT}+\mathbb{Z T}=50 \% \text { in } \mathbf{N S}
$$

(Den Grund für eine solche Ausgeglichenheit zwischen AT und ZT sehen wir im oben explizierten Prinzip der NTS, wonach eine universelle Affinität von komplexen $(M+)$ Kategorien zur komplexeren grammatischen 
Umwelt besteht (vgl. Orešnik 2004). Nebensätze sind demnach in beiden Sprachen, der slowenischen und der deutschen, gleichermaßen beliebte (grammatische) Mikro-Umwelten für das kognitiv komplexe Passiv). Man kann davon ausgehen, dass der Mechanismus der Regelung tilgend eingreifen würde, wenn dieser Grundsatz aus irgendeinem Grund gestört wäre.

6.2.1.2.2 Untersucht wurde auch die Anzahl der gebrauchten Sätze im Allgemeinen und die Zahl der gebrauchten Nebensätze in den AT und den ZT. Folgendes Resultat konnte eruiert werden:

$$
\mathbb{Z T}=\mathrm{M}+=\text { mehr Sätze }(27 \%)+\text { mehr komplexere NS als in AT }
$$

(Durch die allgemein relativ höhere kognitive Markiertheit der ZT (Anspannung, Konzentration, mehr Verarbeitungsoperationen) werden dort mehr markiertere Nebensätze gebraucht. Der Grundsatz,, $\mathbf{M}+$ präferiert $\mathbf{M}+{ }^{66}$ hat sich auch in diesem Fall bestätigt. Dass sich die Waage zu Gunsten der ZT geneigt hat, hat eine pragmatische Begründung (Umstände der Kommunikation, Lampenfieber, Überbrückungszeit, usw.)

Eine pragmatische Gegebenheit, die sich beim Dolmetschen besonders deutlich zeigt, ist die Frequenz der PAUSENGESTALTUNG. Erwartungsgemäß sollte die Dichte der Pausen zu Gunsten der Zieltexte (längere Prozesszeiten, Anspannung usw.) umschlagen, vor allem, wenn diese nicht die für den Translator natürlichere Muttersprache sind. Die statistischen Ergebnisse zeigen folgendes Bild:

ZT $=$ generell um 74\% mehr als AT (Dieses Ergebnis bestätigt die Vorhersage im Sinne der Gebundenheit an die kognitiven Verarbeitungsprozesse))

die wichtigsten I-Träger:

$1 /$ vor $\mathrm{NP}=45 \%$

$2 /$ vor Satzanfang $=30 \%$

$3 /$ vor $\mathbf{V P}=15 \%$

$4 /$ vor sonstigem $=7 \%$

(Zwischen AT und $\mathbb{Z T}$ waren keine relevanten Abweichungen feststellbar, was auf den universellen Charakter der Pausensetzung hindeutet, die sich nach der Relevanz der Wörter im Aktivierungsprozess richtet. Dabei scheinen die NPs als die stärksten Informationsträger zu fungieren, dicht gefolgt vom Satzanfang, wo die Proposition, nachdem sie innerhalb des AT erkannt wurde, in die neue textuelle Umwelt richtig eingebettet werden muss. Fast um die Hälfte weniger wird vor den VPs gezögert (das entsprechende Verbum wird durch die Wahl des Nomens mitaktiviert), und noch einmal halbiert wird die Pausensetzung vor allen anderen Satzkonstituenten.)

Im Bereich der systeminternen Regelung haben sich die oben explizierten Prinzipien der STSN durchweg auch übereinzelsprachlich als geltend ausgewiesen.

Weitere Komponententypen sind noch:

C/ Die Handlung ist die Anpassung an die Umwelt zur Erreichung oder Aufrechterhaltung von Sollzuständen der Umwelt (d.h. aus internen Modellen der 
Umwelt werden erstrebenswerte Zustände der Umwelt gebildet). Diese enge, interaktive Verknüpfung der internalisierten Sollwerte und der tatsächlich erfolgten Reize aus der Umwelt ist auch als die oben bereits erwähnte Sellbstorganisation bekannt und teilt darüber hinaus viele Erklärungsansätze mit dem Konstruktivismus, der Wahrnehmung als Erfindung versteht, wodurch die Kreativität des menschlichen kommunikativen Handelns noch besonders stark hervorgehoben wird). Dadurch wird der Handlungscharakter der sprachlichen und kognitiven Verarbeitung verdeutlicht als die weitere gemeinsame Eigenschaft beider Entitäten. Das erstrebenswerte Resultat ist die KOMMUNIKATIVE TÄTIGKEIT, die eine regulierende, objektivierende und abstrahierende Funktion unter gleichzeitigem Zusammenwirken der Teilprozesse von Handlungen (also von Motiven, Zielen, Folgen von Handlungen und Operationen) zu leisten hat.

Auf diese Weise entsteht die MIKROSTRUKTUR der mit Hilfe der Sprache vollzogenen kommunikativen Tätigkeit. (Langacker 1999; Givon 1995; Chesterman 2000.)

D/ Die Aushandlung setzt die Integration mehrerer KOMMUNIKATOREN mit Hilfe von Handlungen voraus. (Dies kommt bei den T(translatorischen) Prozessen noch in besonderer Weise zum Tragen.) Die Erreichung eines gemeinsamen Zieles durch die Partnerinteraktion ist auf dieser Verarbeitungsebene der ultimative Sollzustand.

Ziele, Situationen und Informationen aus der inneren und äußeren Welt regen zu kooperativen Handlungen an. Somit führen Verständigung und erfolgsorientierte Einstellung zur AUSHANDLUNG bzw. Festlegung des intendierten Themas (Einigung zwischen dem Textproduzenten und dem Textrezipienten bezüglich Thema und Interpretation der Information unter Einbeziehung des kommunikativ relevanten Wissens). (Reickheit-Strohner 1993; Langacker 1991, 1999.) Im Sonderfall der T-Prozesse muss dieses Aushandeln zwischen dem Ausgangstextproduzenten und dem Ausgangstextpublikum stattfinden, des Weiteren zwischen dem Translator und dem Zieltext sowie dem Zieltextpublikum (= maximale Komplexität der Verarbeitungsprozesse). Das Ziel ist die Herstellung relevanter Ähnlichkeiten zwischen dem AT und dem ZT, bzw. die Mimesis des gleichen Textes in eine andere textuelle Umwelt (vgl. Wilss 1988; Chesterman 2000).

Einige interessante Fälle von missglückten Aushandlungen zwischen Translator, Ausgangstext und Zieltext bzw. seinen Adressaten (bei denen sprachliche, zumeist semantische Asymmetrien nicht entsprechend getilgt wurden) möchte ich hier als Beispiele anführen (Als Übersetzer fungierten in den angeführten Beispielen Studenten.):

(1) Wie beurteilen Sie den Preisverfall ... - Kako ocenjujete propad cen ... (Die missglückte Aushandlung beruht auf mangelnder ausgangs- und zielsprachlicher Kompetenz, auf fehlendem Wissen über ausganssprachliche und zielsprachliche Konventionen) 
(2) Sie ist bekennende Muslimin ... - Ona je znana muslimanka (Die missglückte Aushandlung beruht auf mangelnder ausgangs- und zielsprachlicher Kompetenz; es fehlt entsprechendes Wissen über die Kommunikationskonventionen in der Ausgangssprache und auch die Diskurskompetenz in der Zielsprache/Zielkultur weist Mängel auf)

(3) Markenforscher .... über den schleichenden Tod der Marke - Raziskovalec znamke ... o plazeči se smrti blagovne znamke (Die missglückte Aushandlung beruht auf mangelnder ausgangs- und zielsprachlicher Kompetenz; es fehlt entsprechendes Wissen über die Kommunika tionskonventionen in der Ausgangssprache; die Diskurskompetenz in der Zielsprache/Zielkultur weist gravierende Mängel auf)

Die angeführten Beispiele zeigen, dass die entsprechende Aushandlung vor allem bei translatorischen Prozessen die meisten Hindernisse auf den Weg stellt. Diese konzentrieren sich vor allem auf außersprachliche Gegebenheiten wie Diskurskompetenz, Kommunikationskonventionen, Kulturkonventionen, mangelhaftes Weltwissen und Ähnliches.

7.0 Versuchen wir nun noch näher auf die Berührungspunkte zwischen Translation und Kognition einzugehen, indem wir die o.a. Systemkomponenten als Vergleichselemente hinzuziehen. Es wird davon ausgegangen, dass ein derartiger Erklärungsversuch gleichzeitig die Frage zu beantworten vermag, warum eine solche Fragestellung für Untersuchungen von inter- und intralingualen Kommunikationssituationen und Überwindungen von Kommunikationsproblemen überhaupt produktiv sein könnte.

7.1 Die neurolinguistische Realität der Sprache gründet sich auf spezifische neuronale Verknüpfungen (Muster von Synapsen und neuronalen Verknotungen und ihre Aktivierungsmodalitäten), die im Langzeitgedächtnis der Sprachbenutzer angesiedelt sind und somit, wie bereits oben dargelegt, sowohl die universelle Komponente als auch die individuelle Komponente des sprachlichen Systems ausmachen. Jedoch sind diese biologischen Gegebenheiten nicht als fertiges Produkt vorgegeben (mit Ausnahme ihrer Beschaffenheit und Funktionsweise), sondern werden in der Interaktion mit der sozialen und kulturellen Umwelt in ihrer konkreten Ausführung fertiggestellt, jedoch nur um durch das ständige Interagieren der phylo- und ontogenetischen Komponenten ständigem Wandel/An passung an neue Umstände und Bedürfnisse unterworfen zu sein. Die Folge davon ist:

7.2 Zwischen einer Sprache $\mathbf{L} 1(\mathbf{x})$ und einer anderen Sprache $\mathbf{L} 2(\mathbf{x})$ entstehen mehr oder weniger große Abweichungen (biologisch, sozial, kulturell, geschichtlich bedingt). Auf diese Weise verbinden sich in jeder $\mathbb{L}$ 
universelle, prototypische und L-spezifische Komponenten/Merkmale. Dadurch sind Sprachen überhaupt übersetzbar, denn sie teilen sich die universellen Merkmale, die aus vergleichbaren Realitäten generiert werden. Die prototypischen Merkmale verbinden Sprachfamilien und basieren auf ähnlichen bzw. verwandten Realitäten. Letztlich gibt es aber Eigentümlichkeiten, die nur einer einzigen $\mathrm{L}$ angehören und sie von allen anderen in der ihr typischen Weise abheben, weil sie von der ihr eigenen Kultur, von sozialen Konventionen, Normverstößen, Diskurskonventionen usw. hervorgebracht wurde.

7.2.1 Zwischen einer beliebigen Sprache $\mathbf{L} 1(\mathbf{x})$ und einer anderen beliebigen Sprache $\mathbf{L} 2(\mathrm{x})$ liegen unter Umständen auch sozialgruppenbedingte $\mathrm{Ab}$ weichungen (soziolektale Komponenten), des Weiteren interpersonelle Unterschiede (persönliche Erfahrungen, Erlebnisse, Gedanken, Vorstellungen, Gefühle) oder idiosynkratische Komponenten. (Hier finden wir oft die Ursachen für die geringe Kommunikationseffizienz, was im Extremfall sogar zum innersprachlichen Übersetzungsbedarf führen kann.)

7.2.2 Für die T(ranslatorischen) Prozesse sind Konvergenz und transnationale Kommunikation die charakteristischen Züge, basierend auf ähnlichen Überzeugungen aller involvierten Kommunikationssubjekte über die jeweils intendierten Informationen/Propositionen (Chesterman 2000; Wilss 1988; Malmkjaer 1993).

7.2.3 Das $\mathbf{T}$ (System) und das $\mathbf{L}($ System) werden in allen Diskursen von zu erreichenden $\mathbb{Z}$ ielen und zu realisierenden $\mathbb{Z}$ wecken gesteuert, wobei $T$ u.a. dann erfolgreich ist, wenn den kognitiven Gegebenheiten (KS) des AT-Publikums und des $\mathbb{Z T}$ T-Publikums entsprochen werden kann. Dies gelingt durch Überbrückung kognitiver Barrieren, kulturspezifischer Präsuppositionen usw. Aus dem Angeführten kann das folgende Axiom abgeleitet werden

\begin{tabular}{|c|}
\hline TRANSLATIONSPROZESSE \\
\hline L(System) + K(System) + T(System)+ Kulit(System) = Interdependenz \\
\hline $\begin{array}{c}\text { Nurr das Zusammenwirken aller Teilsysteme führt zur erfolgreichen } \\
\text { intersprachlichen/interkulturellen Informationsmittlung }\end{array}$ \\
\hline
\end{tabular}

7.3 Ein komplexes dynamisches Netzwerk kann man aber nicht nur auf dem Makroniveau der T-Prozesse beobachten, sondern auch auf allen Mikroniveaus der mitwirkenden Teilsysteme: $\mathbb{L}$ selbst ist ein Netzwerk von Subsystemen (kognitiven, kulturellen, sozialen ...); $\mathbf{K}($ ultur) ist ein Netzwerk von (Sub)systemen (semiotischen, linguistischen, kulturellen, sozialen). So werden die Komponenten der Systeme unter den Systemen geteilt; sie bilden ihre dynamische Basis, von der aus alle typologischen und spezifischen Merkmale der besprochenen lebendigen Systeme generiert werden. (Systeme 
sind nicht statisch sondern dynamisch, d.h. dem ständigem Wandel unterworfen.) So sind Eigenschaften, wie beispielsweise der mehrfache Gebrauch von gleichen Parametern (Kultur, soziale Konventionen, kommunikative Kompetenz, usw.), sowohl in sprachlichen wie auch in den mit ihnen interagierenden nichtsprachlichen Systemen präsent. Dieser Umstand sorgt dafür, dass die Systeme entsprechend offen und dynamisch sind und dass sie gleichzeitig miteinander kommunizieren können (vgl. Steuerung, Handlung, Aushandlung), um neue kommunikative Werte zu erzeugen:

$\mathbf{T}, \mathbb{L}, \mathbb{K}, \mathbb{K} \mathbf{u}=$ lebendige Systeme, die vergleichbaren Gesetzmäßigkeiten unterliegen; sie breiten sich aus und verändern sich dadurch gleichzeitig (erzeugen Mutationen) = Translation Meme: ,ideas spread, develope and replicate like genes“ (Chesterman 2000)

7.4 Relevante Ähnlichkeiten gibt es demzufolge zwischen den natürlichen Systemen Sprache, Kognition, Kultur und Translation, erzeugt durch ihre gemeinsamen (Sub-) Komponenten:

7.4.1 L ist ein integraler Sub-Bestandteil der mentalen Welt des Benutzers. Durch Interaktion und Koordination wird KOMMUNIKATION als Transfer konzeptualer Inhalte (Informationen) erzeugt. Das wiederum setzt eine enge Korrelation zwischen L-Prozessen und $\mathbb{K}$-Prozessen und SYSTEMEN voraus.

7.4.2 Die K(ognitive)-Infrastruktur dient zur Verarbeitung und Verwendung des Welt - und Situationswissens (Wissen von Objekten und Ereignissen in der physischen Welt, von Strukturen und Konventionen in der sozialen Welt, von eigenen und allgemeinen mentalen Strukturen und Operationen und auch Emotionen, Absichten, Standpunkten und Beziehungen). Auf diese Weise werden einerseits - basierend auf Per zeption und Interpretation der Realität - Urteile generiert, andererseits basierend auf kreativer Imagination - Fiktion. RELEVANTE ÄHNLICHKEITEN mit T-Prozessen sind offensichtlich.

7.4.3 Die Ähnlichkeitsbeziehungen zwischen $\mathbf{L} / \mathbf{K}$ und $\mathbf{G}$ (Grammatik) basieren auch auf Kompatibilität und Konvergenz, was im Einzelnen etwa folgendermaßen funktioniert: Das $\mathbf{K S}$ setzt das GS (grammatisches System) darüber in Kenntnis, welche konzeptuellen Elemente (Weltwissen) für bestimmte Kommunikationszwecke lexikalisiert werden müssen.

7.4.3.1 Das GS hat Rückwirkung auf die Identifikation und Interpretation von konzeptuellen Elementen. Somit wird deutlich, dass die G-Kategorien als SEMANTISCHE OPERATOREN fungieren, wodurch der KREISLAUF 
dieser natürlichen Systeme ermöglicht wird. Durch Veränderung und Anpassung entsteht ein wechselwirkendes, lebendiges, der Kreativität fähiges Gebilde: die menschliche Kommunikation (= Koordination des gesamten Wissenserwerbs und Versorgung aller Typen der vom Wissen aktivierten Handlungen). Sprachliches Wissen und nichtsprachliches Wissen werden dynamisch gekoppelt, was eigentlich die Kohärenz des gesamten menschlichen Verhaltens ausmacht.

Einige Bespiele aus den von Studenten produzierten Übersetzungsversuchen sollen die enge Verbundenheit zwischen Grammatik und Konzeption veranschaulichen und gleichzeitig die negativen Folgen von Unzulänglichkeiten auf dem einen oder anderen Gebiet unterstreichen:

(4) Manfred Schmidt ... warnt Markenunternehmen ... - Manfred Schmidt je posvaril podjetnike (mangelnde AS-Kompetenz, unzulänglicher Grammatik-Transfer, fehlendes fachsprachliches Spezialwissen > fraglicher Kommunikationserfolg)

(5) Dann wird draußen noch immer beworben, was drinnen schon wegrationalisiert wurde. - Na trgu tako podjetja navzven konkurirajo s stvarmi, katere pa so že naredili odvečne pri racionalizaciji. (mangelnde AS/ZS-Kompetenz, mangelnde K-Kompetenz; unzulänglicher Grammatik Transfer, einschließlich Stilebenen)

(6) Cerkev je bila $v$ siloviti krizi - die Kirche ist in heftige Krise geraten (Perfekt statt Prät. = temporale Inkompetenz.; perfektiv statt imperf. = mangelhaftes Wissen im Bereich des Aspektes > fraglicher Kommu nikationserfolg, vor allem textsortenspezifisch)

(7) Ki naj bi bila popravila - die wieder in Ordnung gebracht haben konnte (mangelhaftes grammatisches Wissen im A-T-M-Bereich > fraglicher Kommunikationserfolg)

(8) je očka mirno odracal - watschelte Vater ruhig (imperf. statt perf. = mangelhaftes grammatisches Wissen im A-T-Bereich > fraglicher Kommunikationserfolg, vor allem textsortenspezifisch)

(9) $\quad v$ nekaterih pokrajinah še danes pravijo - in einigen Landschaften wird noch heute gesagt (perf. statt imperf. = mangelhafes grammatisches Wissen im A-T-Bereich > fraglicher Kommunikationserfolg, vor allem textsortenspezifisch)

(10) ...vom Genfer Institut... - iz Instituta v Genovi (mangelnde ZS-Grammatik, unzulängliches Wissen über Übersetzungsnormen) 
7.4.4 Kognitive Strukturen sind ihrerseits, genau wie die Sprache, unzertrennlich mit der Umwelt verbunden, denn sie werden durch die strukturellen Eigenschaften des Verhaltenssystems, in welchem sie kodiert sind, kanalisiert (= Einfluss der soziokulturellen Umwelt $(\mathbf{K u})$ auf $\mathbf{L}, \mathbf{K}$ und folglich auch auf die T-Prozesse, die eigentlich als dynamische Summe der vorerwähnten Entitäten und noch einiges mehr aufzufassen sind. In diesem Umstand liegt nämlich die Begründung für das Entstehen der kulturell und sozial bedingten Asymmetrien zwischen unterschiedlichen L/K-Systemen.

Einige Beispiele aus der Übersetzungspraxis von Studenten sollen die $\mathrm{Ku} / \mathrm{K}$-induzierten, nur mit guten translatorischen Erfahrungen überbrückbaren konzeptuellen Hindernisse veranschaulichen. (Neben dem sprachlichen Wissen ist für erfolgreiche T-Prozesse auch jenes über die kulturellen Eigenheiten und Konventionen und die jeweils ritualisierten Kommunikationsmuster und Diskurskonventionen erforderlich):

(11) »Nur abspecken, wo Fett da ist« - Shujšati samo, kjer je maščoba. (mangelnde kommunikative Kompetenz/Konventionen in der ZS; fehlende Diskurskompetenz in der AS und ZS; nicht erfolgter Sprach-Kulturtransfer)

(12) Wie beurteilen Sie den Preisverfall ... - Kako ocenjujete propad cen ... (mangelnde ZS -Kompetenz/Konventionen; unzulängliche Diskurskompetenz in der Zielkultur)

(13) ... dass sie oftmals persönlich wegen ihres Engagements bedroht worden ist. ... - ... da je bila pogosto osebno ogrožena zaradi svoje vpletenosti (mangelnde ZS -Kompetenz/Konventionen; unzulängliche Diskurskompetenz in der Zielkultur)

(14) ... um gleich hohe Ergebnisse zu erzielen ... - če želimo dobiti enako velike rezultate (mangelnde kommunikative Kompetenz/Konventionen in der ZS; nicht erfolgter Sprach-Kulturtransfer)

\subsection{Schlusswort und Zusammenfassung}

Die Parallelitäten und unabdingbaren Wechselwirkungen zwischen den natürlichen Systemen Sprache, Kognition, Kultur und Translation kann man wie folgt zusammenfassen:

8.1 Alle verbindet der Umstand, dass sie offene, dynamische und natürliche Systeme sind, ausgewiesen durch Eigenschaften von natürlichen Systemen (=Vererbung von genetischem Material; Ausdehnung und Wechselwirkung mit anderen Systemen; Metamorphosen, Mutationen). 
8.2 Eine weitere gemeinsame Eigenschaft ist die Kommunikabilität (= Neigung zur Kommunikationsoptimierung (Mitberücksichtigung von sozialen Normen; Aushandlung als Überwindung von kognitiven Hindernissen usw.).

8.3 Die natürlichen Systeme $\mathbf{L}, \mathbb{K}, \mathbf{K u}$ und $\mathbf{T}$ wirken ineinander, ergänzen sich. Nur als Gesamtheit sind sie kommunikationsrelevant. Unter Punkt 7.4.4 wurde jedoch erwähnt, dass die T- Prozesse nicht nur die dynamische Summe von $K / L$ und $K u$ Systemen sind, sondern noch um einiges mehr. Das Letztere soll nun noch kurz expliziert werden.

8.3.1 Integraler Bestandteil des T-Systems sind nämlich neben den o.a. auch weitere außersprachlich verankerte Entitäten wie Loyalität, Integrität und andere ethische, kommunikative, stilistische u.a. Normen (dynamischer Aspekt) oder der systematische Bruch dieser Normen, bekannt als Stilem. Weitere Merkmale sind zudem noch die Relationalität zwischen AT und $\mathbb{Z T}$ (in Abhängigkeit von Texttyp, Anforderungen des Kunden, Intentionen des AT/Produzenten, Erwartungen des ZT/Rezipienten), die Art der Adaptiertheit an die ZT/Kultur (= Berücksichtigung der Kultureme als Elemente/Bausteine des natürlichen Systems Kultur), AT und ZT/ Relation im Sinne von Hinzufügen oder Auslassen von Informationen (Textausdehnung in neue kommunikative Welten).

8.3.2 Alle erwähnten natürlichen Systeme, besonders aber das T-System zeichnen sich durch den Einsatz von nichtsprachlichen (auch prosodischen) Mitteln aus, was bei T-Prozessen noch besonders stark zum Tragen kommt, womit ihre ausgesprochen hohe Komplexität ausgewiesen wird.

Wenn eine der zum Erfolg führenden Komponenten der T-Prozesse aussetzt oder Mängel aufweist, können in Extremfällen fatale Folgen entstehen. Nicht gerade fatal, dennoch überlegenswert ist das folgende Beispiel:

„Mejne prehod je dovoljen: z izkaznici za „mali mejni prehod“. Z potnim listom ali $z$ osebni izkaznici za avstrijske drzavljane in tudi za slowenskedrzavljane in tudi za taksne osebe, katere nimajo vise, peske ljudi, kateri se vozijo z bicikelnom in z malim motornim vozil do $125 \mathrm{ccm}$."

(So lautet die Aufschrift auf einer offiziellen Tafel, angebracht an einem Grenzübergang für den kleinen Grenzverkehr zwischen Österreich und Slowenien. Eine qualitätsbewusste translatorische Lösung könnte in diesem Fall positive diplomatische Konsequenzen haben ...)

8.4 Ähnliche Beschreibungs- und Erklärungsmodelle des natürlichen Systems Sprache werden auch von der NT angeboten. Die NT wie auch die STSN gehen davon aus, dass sprachliche und nichtsprachliche Elemente 
wechselwirkend offene, dynamische sprachliche/kommunikative Systeme ohne rigide Abgrenzungen zwischen ihren Bestandteilen (Paradigmen, Modulen) bilden. Dadurch sind sie von Fall zu Fall einzigartig und somit finalistisch (= abhängig von situativen, kulturbedingten und anderen eine konkrete Kommunikationssituation prägenden Merkmalen). Gleichzeitig berührt die Tatsache, dass sie $(\mathbf{L}, \mathbf{K}, \mathbf{K u}$ und $\mathbf{T})$ gleichen generellen Gesetzmäßigkeiten unterliegen, ihren universellen Charakter, wodurch sowohl Kommunikation als auch Übersetzbarkeit möglich gemacht werden.

8.5 Auch das als Regelung bekannte Korrektiv, wodurch Markiertheit, Frequenz, Systemangemessenheit etc. als universelle Prinzipien übereinzelsprachlich wirken, konnte in allen im Aufsatz behandelten natürlichen Systemen als wirksamer Mechanismus konstatiert werden.

8.6 Konkrete Berührungspunkte zwischen Kognition, Sprache, Kultur und letztlich Translation mit den Erkenntnissen der STSN wurden im Rahmen der hier diskutierten Pilotstudie lediglich für die Systemkomponente der Regelung näher erläutert. Die Ergebnisse bestätigten die Arbeitshypothese, wonach in allen Systemen die Mikroverhältnisse durch die Regelung im Gleichgewicht gehalten werden. Im Konkretfall wurden die Markiertheitsund Frequenzverhältnisse zwischen $A T$ und $Z T$ als stabile sprach- und kulturübergreifende Universalien ausgewiesen. Weitere Untersuchungen sollen auch auf andere Komponenten des kognitiven Systems und auf fehlende oder bestehende Parallelitäten zur L im Sinne der Erklärungspotenziale der STSN näher eingehen.

8.7 Abschließend bleibt festzuhalten, dass Sprache, Kognition, Kultur und Translation komplexe systembezogene Tätigkeiten sind, rekurrent und ineinandergreifend, vom Menschen interaktiv, multidisziplinär (selbst)konstruiert. Sinngemäß fasst der vom Konstruktivismus hervorgebrachte Satz "Sprache ist nicht, sie geschieht" das beobachtete sprachliche, kulturelle, kognitive und translatorische Geschehen/Handeln des Menschen zusammen. 


\section{Literatur:}

ButzKamm, W. (1989). Psycholinguistik des Fremdsprachenunterrichts. Francke Verlag Tübingen.

Chesterman, A (2000). Memes of Translation. John Benjamins, Amsterdam, Philadelphia

Dressler, W.U.; Mayerthalet, W.; Panagl, O.; Wurzel, U. (1987). Leitmotifs in natural Morphology. Amsterdam. John Benjamins.

DRESSLER, W.U. (1990). »The cognitive perspective of »naturalist« linguistic models«. In: Cognitive Linguistics $1-1 ; 75-78$.

Grvon, T. (1995). Functionalism and Grammar. Amsterdam. John Benjamins.

Hatch Marcussen, E. (1983). Psicholinguistics. A second Language Perspective. Cambridge. Newbury House Publishers.

KARPF, A.(1990). Selbstorganisationsprozesse in der sprachlichen Ontogenese: Erst- und Fremdsprache(n). Tübingen. Narr.

KARPF, A. \& Dringel-Techt. (1995). »The Role of Morphological Naturalness in Second Language Development«. In: Dressler, W. U. \& Burani, C. (eds.) (1995), Crossdisciplinary Approaches to Morphology. Wien: Verlag der Österreichischen Akademie der Wissenschaften. 131-147.

Kess, Joseph F. (1992). Psicholinguistics. Psychology, Linguistics ans the Study of Natural Language. Amsterdam/Philadelphia. John Benjamins.

LANGACKER, W. Roland (1991): Concept, Image, and Symbol. Mouton de Gruyter, Berlin, New York.

LANGACKER, W. Roland (1999): Grammar and Conceptualization. Mouton de Gruyter, Berlin, New York.

MALMKJAER, K. (1993). "Underpinning translation theory". In: Target 5(2): 133 - 148.

MAYerthaler, W., Morphological Naturalness, Karoma, Ann Arbor. 1985.

OREŠNIK, J.(1999). Krepke in šibke dvojnice $v$ skladnji. Ljubljana. Slovenska akademija znanosti in umetnosti.

OREŠNIK, J. (1994). Slovenski glagolski vid in univerzalna slovnica. Ljubljana: SAZU.

OREŠNIK, J. (2000). "Naturalness. The scale formats $>$ sem (+/-A, -A) and > sem (+/-A, +A). In: Linguistica 40, 237-62. Orešnik J. (2000). Naturalness: Some Slovenian (morpho)syntactic examples. Slovenski jezik Slovene Linguistic Studies 3, 2001a, 3-31.

OREŠNIK, J. (2003). "Naturalness. Some Norwegian (morpho)syntactic examples". In: Norsk Lingvistik Tidsskrift, $21,57-70$.

OREŠNIK, J. (2004). Naturalness in (morpho)syntax: English examples $=$ Jezikovna naravnost $v$ (obliko)skladnji: angleški zgledi. (Dela 61). Ljubljana: Slovenska akademija znanosti in umetnosti.

PETRI, H.L.; Mishkin, M., (1994). »Behaviorism, cognitivism and the neuropsychology of memory«. In: American Scientist, 92.

Rickheit G., Strohner H.(1993). Grundlagen der kognitiven Sprachverarbeitung. Tübingen, Basel; Francke.

Teržan-Kopecky, K. (1997). »Die Typologie der Abfolge von Spracherwerbsprozessen«. In: Sprachtypologie und Universalienforschung. Berlin. 50, Nr.. 4,348-357.

Teržan-Kopecky K. (1998). „Zur natürlichen Syntax“. In:Sammelband des II. internationalen Symposions zur Natürlichkeitstheorie. Redaktion: Karmen Teržan Kopecky. Maribor.

Teržan-Kopecky, K. (2001). Psihološke dimenzije jezikovnih ravnanj. Maribor, Pedagoška fakulteta.

Wilss, W. (1988). Kognition und Übersetzen. Zur Theorie und Praxis der menschlichen und der maschinellen Übersetzung. Tübingen: Niemeyer. 
Osrednja tema prispevka so naravni sistemi oz. teorija o kognitivnem sistemu (njegove komponente, dinamika), kot jo predlagajo sodobne spoznavne teorije (Langacker, 1999, RickheitStrohner 1993 in drugi). Teorija o ustroju in aktivnosti spoznavnega sistema pri človeku je upo rabljena kot temelj za preverjanje skupnih (naravnih) lastnosti, ki si jih slednji deli še z drugimi naravnimi sistemi, zlasti z jezikom, ki je v prispevku obravnavan v posebnih okoliščinah medkulturnega sporazumevanja (prevajanje/tolmačenje slo $\leftrightarrow$ ne).

Prikazan je tudi proces konfrontacije dveh teoretskih pristopov, ki najdeta skupno podmeno $\mathrm{v}$ opisovanju mehanizmov spoznavne (in/ali jezikovne) predelave informacij in kasnejšega ravnanja $\mathrm{z}$ njimi (sporočanje/sporazumevanje). Pri prvem gre za že omenjeno spoznavno teorijo, pri drugem pa za teorijo naravnosti, natančneje slovensko teorijo jezikovne naravnosti (STJN).

$\mathrm{Na}$ mikrojezikovnem nivoju medjezikovne stičnosti (slo-nem prevodna besedila) je prikazano delovanje sistemske komponente uravnavanje, ki med drugim skrbi za to, da jezikovno univerzalni pojavi, kot so zaznamovanost (spoznavna in jezikovna) in pogostnost rabe delujejo sorazmerno neodvisno od značilnosti konkretnih sporočevalnih okoliščin (doživljajskega okvirja).

V kontekstu medjezikovnega/medkulturnega pretoka jezikovnih informacij so seveda v ospredju še druge sistemske komponente, ki pa so se izkazale kot izjemno odvisne od širšega in ožjega doživljajskega okvirja (kulturemov, sporočevalnih modelov in konvencij, idr.). 